\title{
Metalogical Remarks on Induction
}

\author{
Jan Woleński ${ }^{1,2}$
}

Received: 30 April 2021 / Accepted: 9 June 2021 / Published online: 8 July 2021

(c) The Author(s) 2021

\begin{abstract}
The problem of induction belongs to the most controversial issues in philosophy of science. If induction is understood widely, it covers every fallible inference, that is, such that its conclusion is not logically entailed by its premises. This paper analyses so-called reductive induction, that is, reasoning in which premises follow from the conclusion, but the reverse relation does not hold. Two issues are taken into account, namely the definition of reductive inference and its justification. The analysis proposed in the paper employs metalogical tools. The author agrees with the view that a quantitative account of degree of confirmation for universal theories via logical probability is problematic. However, prospect for a qualitative approach look as more promising. Using the construction of maximally consistent sets allows to distinguish good and worthless induction as well as shows how to understand induction in a semantic way. A closer analysis of deductivism in the theory of justification shows that it is a hidden inductivism.
\end{abstract}

Keywords Reduction · Probability · Statistics · Confirmation · Inductivism · Deductivism

\section{Introduction}

Every contemporary general compendium in the philosophy of science (see Newell-Smith 2000; Machamer and Silberstein 2002; Kuipers 2007; Psillos 2013; Humphreys 2016) has a chapter on induction. Moreover, inductive procedures are analyzed in separate parts of more specialized anthologies, particularly devoted to probability and statistics (see Kyburg 1974; Bandyopadhyay and Forster 2011;

Jan Woleński

jan.wolenski@if.uj.edu.pl

1 University of Information, Technology and Management, Rzeszow, Mickiewicza 26, 34-200 Sucha Beskidzka, Poland

2 Jagiellonian University, Kraków, Poland 
Hájek and Hitchcock 2016). According to my knowledge, Inductive logic became the subject of three monographs published in the last 6 years-they are Paris and Vencovská (2015), Williamson (2016), Johnson (2017). ${ }^{1}$ These facts provide a good preliminary documentation that the problem of induction further belongs to the vital problems in the philosophy of science. Perhaps it is not so hotly discussed as when the Carnap-Popper controversy achieved its peak in the 1950s and 1960s (see Michalos 1971), but still arouses serious interests. One thing remains stable, namely that everything is controversial around induction. What is inductive logic? Since inductive logic cannot be reduces to deductive one, how to codify rules inductive inferences? How to evaluate the correctness, if any, of inductive reasoning? Is induction relevant for science? If we positively answer to the former question, is induction a method of discovery or a method of justification? Inductivism positively answers to the penultimate question, that is, argues that inductive procedures are applied in the real physics, for instance, that general laws are inferred by induction from premises concerning particular facts or general hypotheses are confirmed (justified) by deducing true consequences from them as assumptions. I am not very much interested in the role of induction in working science (see papers in Cohen and Hesse 1980) for a discussion; in fact, almost every work on inductive procedures address to this question, directly or indirectly). I consider my analytical task as mainly epistemological. We have a kind of reasoning which is fallible by definition, that is, it can start with true premises and result in false conclusions. Yet fallible inferences are or might be regarded as "good" (confirming truth of conclusions) or "bad" (failing to confirm). Now the question is how to distinguish both kinds of induction and how to justify induction at all. I will apply to the analysis of both questions tools derived from metalogic. Due to this aim, my treatment of various problems, historical as well as substantial, is deliberately general and simplified in many points.

\section{What is Induction?-The First Approximation}

Since there many approaches to induction and inductive logic (see Gabbay et al. 2011 for a survey), I will not give a definition of induction at the beginning of my analysis, but proceed step by step. So-called enumerative induction is the first approximation. It falls under the scheme (the symbol $f^{\text {ind }}$ can be read "entails inductively")

$$
P a_{1}, \ldots, P a_{n} \vdash^{i n d} \forall x P x,
$$

where $P a_{1}, \ldots, P a_{n}$ are singular sentences, that is, about individual object denoted by proper names (individual constants) $a_{1}, \ldots, a_{n}$. In words, we have that if items $a_{1}, \ldots, a_{n}$ (for simplicity, I do not distinguish individual constants and their denotata by separate symbols) satisfy the condition $P$, than $\forall x P x$, holds. In order to make

\footnotetext{
1 Some earlier writings on induction will be quoted in what follows. I note that I do not pretend to mention even a moderate number or relevant works on induction.
} 
metalogical remarks easier, I change the notation by replacing $f^{\text {ind }}$ by the symbol $C n^{\text {ind }}$ (the inductive consequence). It is justified by the dependence (I use the simplest case) $\left.A\right|^{\text {ind }} B$ iff (if and only if) $B \in C n^{i n d}\{A\}$ analogous to the definition $A+$ $B$ iff $B \in C n\{B\} .{ }^{2}$ It is convenient to consider deduction at this point as a special case of induction. Thus, we have

$$
\begin{gathered}
\forall x P x \in C n\left\{P a_{1}, \ldots, P a_{n}\right\} \Rightarrow \forall x P x \in C n^{i n d}\left\{P a_{1}, \ldots, P a_{n}\right\} \\
A \in C n X \Rightarrow A \in C n^{i n d} X
\end{gathered}
$$

(2a) is related to (1), but (2b) displays the general case. This convention (I do not propose it as a general definition) allows to consider so-called complete induction (the scope of the universal quantifier is exhausted by the objects $a_{l}, \ldots, a_{n}$ ) as a species of deductive inference. If we intend to differentiate induction and deduction, we can stipulate that (I consider the case related to (1) only)

$$
\forall x P x \in C n^{\text {sind }}\left\{P a_{1}, \ldots, P a_{n}\right\} \wedge \neg\left(\forall x P x \in C n\left\{P a_{1}, \ldots, P a_{n}\right\},\right.
$$

where the symbol $C n^{\text {sind }}$ refers to induction in the strict sense. ${ }^{3}$

If we specify (2) by stipulating

$$
B \in C n^{\text {sind }}\left\{A_{1}, \ldots, A_{n}\right\}, \text { provided that for any } A_{k}(1 \leq k \geq n), A_{k} \in C n\{B\},
$$

we obtain the scheme of reductive (abductive; I neglect a closer comparison of induction and abduction) inference. ${ }^{4}$ According to (4), every premise of reduction is deductively entailed by its conclusion. Enumerative induction automatically falls under the condition (4), because for any $k(1 \leq k \geq n), A_{k} \in C n\{\forall x\}$. We can say that (2a) displays a traditional intuition that induction proceeds from the particular to the general, but complete enumerative induction account the old understanding of deduction as inference from the particular to the general.

\section{A More General Approach}

However, (4) appears as too weak as a definition of inductive reasoning as fallible. It does not cover statistical induction, Mill's canons of eliminative induction and analogy, that is, cases in which the deductive connection holds neither from premises to conclusion not in the reverse direction. This suggests a more general scheme, namely

\footnotetext{
${ }^{2}$ I consider induction in the context of justification only, not in the context of discovery.

${ }^{3}$ Although the settings (2) and (3) lead to some problems (see below), most my further remarks concern induction in the strict sense.

${ }^{4}$ The distinction of deductive and inductive inferences was very popular in Poland (see Łukasiewicz 1912, Czeżowski 2000), and Woleński (1991) for a general survey.
} 
$B \in C n^{\text {sind }}\left\{A_{1}, \ldots, A_{n}\right\}$ iff the premises $A_{1}, \ldots, A_{n}$ make probable that $B$.

An immediate problem related to (5) consists the concept of probability in this context. Even if we neglect various interpretations in mathematics or the foundations of statistics, there remains the question labelled as "induction and probability” (see for instance Lakatos 1968, Kyburg 1970, Jeffrey 1992, Stegmüller 1973, Lenzen 1974, Hacking 2002, Gabbay et al. 2011 for surveys and proposals how to do with this question) - the crucial issue consist in defining probability as a logical relation holding between $A_{1}, \ldots, A_{n}$ and $\mathrm{B}$, that is, attributed to sentences, not to events. ${ }^{5}$ If one decides to skip an appeal to probability, he or she can adopt

$$
B \in C n^{\text {sind }}\left\{A_{1}, \ldots, A_{n}\right\} \text { is an inductive inference iff it is fallible, }
$$

as a general definition of induction. Unfortunately, (6) does not specifies properties of $C n^{\text {sind d }}$ except saying that it is not deductive. This approach leads to difficulties illustrated by the following examples. Consider the inferences (I use a simplified formulation in 8)

The speed of light in vacuum is not constant $\in C n^{\text {sind }}\{$ the battle of Waterloo occurred in 1815$\}$;

Principles quantum mechanics in the Copenhagen interpretation $\in C n^{\text {sind }}\{$ the uncertainty principle $\}$.

The first inference is fallible and there is no substantial link between true premise and false conclusion, but (8) also not deductive (we can imagine a model in which the Heisenberg principle holds, but the Copenhagen interpretation fails. Now, (7) provides an example of a "bad" induction, that is, not justifying the conclusion, but (8) offers a case of "good" one, that is, justificatory.

Kazimierz Ajdukiewicz (see Ajdukiewicz 1955) proposed the division of inferences into three groups (i) deductive; (ii) probabilistic; (iii) worthless. The second case covers fallible inferences, but making the conclusion probable ( 8 might serve as an example). Consequently, a worthless inference is neither deductive nor probabilistic (as in the example (7). Additionally, we have the intuition that a valuable fallible inference is a mental process leading to assertion of the conclusion relatively to asserted premises, provided that the degree of conclusion-assertion is higher that the degree of premises-assertion. ${ }^{6}$ In this framework, deductive inferences can be characterized as such in which the degree of conclusion-assertion does not exceed the degree of premises-assertion. We can formalize this proposal by (the notation $\mathrm{Ass}_{\mathrm{r}}$ means "asserted in the degree $r$ ")

$$
B \in C n^{\text {sind }}\left\{A_{1}, \ldots, A_{n}\right\} \wedge \operatorname{Ass}_{\mathrm{s}}(B) \wedge \operatorname{Ass}_{\mathrm{r}}\left(A_{1}, \ldots, A_{n}\right) \wedge r \geq s-\text { the case of deduction; }
$$

\footnotetext{
5 I will return to this question in what follows.

${ }^{6}$ One might observe that a worthless reasoning does not deserve to be called an inference, but it is merely a terminological problem.
} 
$B \in C n^{\text {sind }}\left\{A_{1}, \ldots, A_{n}\right\} \wedge \operatorname{Ass}_{\mathrm{s}}(B) \wedge \operatorname{Ass}_{\mathrm{r}}\left(A_{1}, \ldots, A_{n}\right) \wedge r>s$-the case of probabilistic inductive inference

The last setting does not employ the concept of truth. Since the application of the notion to deduction follows from the definition of this kind of inference, it remains (9b). The simplest way is to propose ( $A \in \mathbf{V E R}$ means " $A$ is true")

$$
\left.B \in C n^{\text {sind }}\left\{A_{1}, \ldots, A_{n}\right\} \wedge B \in \mathbf{V E R} \wedge \forall A_{k(1 \leq k \geq n)} A_{k} \in \mathbf{V E R}\right) .
$$

However, the degrees $r$ and $s$ disappear-the probabilistic element is hidden in $\mathrm{Cn}^{\text {sind }}$. Eventually, one might propose

$$
B \in C n^{\text {sind }}\left\{A_{1}, \ldots, A_{n}\right\} \wedge \operatorname{Ass}_{\mathrm{r}}(B \in \mathbf{V E R}) \wedge \forall A_{k(1 \leq k \geq n)} \operatorname{Ass}_{\mathrm{s}}\left(A_{k} \in \mathbf{V E R}\right) \wedge r>s .
$$

In words, an inference is probabilistically inductive, if its conclusion asserted in the degree $s$ as true (note that it does not mean-asserted as true in the degree $s$ ) on the base of premises asserted in the degree $r$ and $r<s$. Yet this formulation is unclear due to the lack of a definition of asserting in a degree $r$ (or $s$ ) as trueit is also unclear whether asserting, to a certain degree, a sentence from a given set requires that every sentence from this set is asserted to the same degree $n$ (this remark applies to $9 \mathrm{~b}$ as well). The main problem of induction, directly or indirectly suggested by the formulas (1)-(11) lead to the question whether the truth of premises of a strict inductive inference guarantees the truth of its conclusion.

Hume famously argued for a negative answer to the above question (see Hume (1748/2000, p. 24-25, p. 31).

All the objects of human reason or enquiry may naturally be divided into two kinds to wit, Relations of Ideas and Matters of Facts. Of the first kind are Sciences of Geometry, Algebra and Arithmetic; and in short, every affirmation, which is either intuitively or demonstratively certain. [...]. That three times five is equal to the half of thirty, expresses the relation between these numbers. Propositions of this kind are discoverable by the mere operations of thought, without dependence on what is any where existent in the universe. [...]. Matters of facts, which are the second objects of human reason, are not ascertained in the same manner; not is our evidence of their truth, however, great of a like, nature with foregoing. The contrary of every matter of facts is still possible; because we can never infer a contradiction [...]. All reasonings concerning matter of fact seem to be founded of the relation of Cause and Effect. By means of that relation alone we can go beyond the evidence of our memory and senses. [..]. I shall venture, as a general proposition, which admits of no exception, that the knowledge of this relation is not, in any instance, attained by reasonings a priori; but arises entirely from experience, when we find that any particular objects, are conjoined with each other. [...]. We have said that, that all arguments concerning existence are founded on the relation of cause and effect, that our knowledge of this relation is derived entirely from experience; and that all our experimental conclusions proceed upon the supposition that, that the future will be conformable with the past. The endeavour, therefore, the proof that of this last supposition by probable arguments, or arguments regarding existence, must be evidently going in a circle, and taking that for granted, which is the very point in question. 
This passage raises two issues, both previously mentioned (I use the present terminology), firstly, the contrast between deduction and induction, and, secondly, the problem of justification of induction. Although Hume understood induction as nondemonstrative inference, he considered this kind of reasoning as occurring in science and daily life, base on habits and customs. Consequently, induction has no logical justification, but practical one.

As I noted at the end of Introduction, my methodology consists in employing metalogical devices in analyzing of induction. At first, let me compare strict (empirical) enumerative induction with mathematical one. The latter falls under the following scheme ( $\mathbf{N}$ - the set of natural numbers)

$$
\left.P a_{1} \wedge \forall k \in \mathbf{N}\left(P a_{k} \Rightarrow P a_{k+1}\right)\right) \vdash \forall n \in \mathbf{N}\left(P a_{n}\right) .
$$

As the notation indicates, (12) appears as a scheme of a deductive inference. In order to prove the conclusion it is sufficient to show that $P a_{1}$ (the initial step) and that $\forall k \in \mathbf{N}\left(P a_{k} \Rightarrow P a_{k+1}\right)$ (the inductive step). This procedure is lacking in the case of empirical induction. Suppose that the initial step is given. The difficulty concerns the inductive step, because, except complete induction, we have no purely logical justification for the inductive step, that is, the assertion $\forall k \in \mathbf{N}\left(P a_{k} \Rightarrow P a_{k+1}\right)$ as valid without any exception. The principles of the uniformity (stability) of nature or causality as necessary relation, both observed by Hume, as well as the rule of limited diversity, could serve as assumptions for proving the inductive step, but they raise doubts as leading to vicious circle or infinite regress. The basic defect of such solutions consists in the fact that strict empirical induction was frequently fallible. It seems, and it is an interesting observation, attempts to justify induction as reported by Hume and his followers presuppose still one additional condition, namely that legitimate (good) inductive inferences should always proceed from true premises to true conclusions. However, this supposition appears as dubious, because an essential feature of induction consists in its fallibility, directly leading to the problem of how good inductions differ from bad ones.

Since (12) (and former formulas belong to metalogic), employing metalogical devices to analyze inductive inference appears as a reasonable strategy. Clearly, since the operation $\mathrm{Cn}$ characterizes deduction, introducing $\mathrm{Cn}^{\text {sind }}$ might be considered as the first step. The starting intuition is as follow. If we at our disposal true statements confirming a hypothesis $H$, it means that we possess reasons for its truth as well. In other words, induction works, when we cannot deductively prove that $H$ is true, but there exist witnesses that the hypothesis in question deserves to be qualified as true. Consequently, the most important task for analysis of inductive inference consists in providing rules elaborating of how the growth of supporting data for $H$, increases its justification as true. A natural response the above intuitive postulate seems to be as follows. If we are not able to give a deductive proof that $H$ is true, relative to an initial collection of empirical data, assume that a good induction attributes to $H$ a "sufficient degree" of probability and that new data confirming 
this hypothesis "increase" this measure. The quotes in the preceding sentence indicated that the issue is controversial. Roughly speaking, we have two approaches to the logic of induction, namely statistical and non-statistical. ${ }^{7}$ The first approach (see Paris and Vencovská 2015, Williamson 2016) considers induction as a kind of statistical inference. Its universality depends on a controversial claim that everything in the actual world is subjected to statistical uniformities. ${ }^{8}$ The rejection of this postulate results in limiting the related solution to statistical phenomena. In fact, the inductive logic conceived in such a way becomes a branch of applied statistics-looking at works in this direction convinces that their logical content is rather poor. The second appears in at least (to my knowledge) two versions. One consists in using game and decision theory (see Ajdukiewicz 1958, 1974; Maher 1993) and defining the betting on theories (hypotheses) dependent on evaluation of profits and losses resulting from acceptance or rejection of a given theory. A different approach was proposed by Carnap (1950). He assumed that the abstract mathematical concept of probability has two interpretations, statistical and logical. The latter requires to define the function $c(H, e)$ to be read "the confirmation of a hypothesis $H$, relatively to an evidence $e$ " in a way that it satisfies the axioms of mathematical probability theory. In particular, we have that $c(H, e)=r$, where $0 \leq r \geq 1$-thus $r$ expresses a measure of logical probability of $H$, relatively to $e$. If $r=0$, then $e$ contradicts $H$; if $r=1$, then $H \in C n\{e\}$ (the notation is simplified-note that the second formula justifies 6). ${ }^{9}$ Another reading of $r$ is "the degree of logical probability, if $c(H, e)$ ". Looking at $r$ from another perspective, it expresses the degree of inductive support for $H$. The main problem of Carnap's construction is that the initial $r$ for strictly universal hypotheses is equal to 0 and cannot increase by collecting new evidence. My task consists in showing that this situation cannot be improved and we must stay with the qualitative analysis of confirmation. Before going further I would make a methodological remark. One might observe that the logic of induction, whatever it is, has nothing common with the real scientific practice. I do not enter into this question, because, as I noted in Sect. 1, my interest in the problem of induction is epistemological and, except a general statement that they appeal to empirical evidence, does not consists in reporting how scientists justify their theories.

\section{A Metalogical Analysis of Reductive Induction}

I choose Carnap's proposal for further analysis. It requires embedding it into the terminology of the present paper. I assume that, inductive confirmation acts on empirical theories, that is, deductive systems, that is, sets of sentences satisfying the

\footnotetext{
7 In further considerations, I will omit analogical reasoning (see Pietarinen 1972 for an analysis of analogy in the context of induction) and eliminative induction (this kind of reasoning is rarely mentioned in the contemporary writings).

${ }^{8}$ Eventually, one can try to generalize the Bayesian approach to non-statistical hypotheses, but his way has some difficulties with a priori probabilities (see Shafer 1976).

9 I omit details of axiomatization of $c(H, e)$.
} 
condition that if $T$ is a theory, then $T=C n T$. If we add that $T$ is based on hypotheses $H_{1}, \ldots, H_{n}$ as axioms, confirmation of $T$ can be reduced to inductive support of the axiomatic base of $T$. We define

A set $X$ of sentences is a confirmation of a theory $T$ iff the following conditions hold

$X$ is a finite set, that is, $X=\left\{A_{1}, \ldots, A_{n}\right\}$-elements of $X$ are called confirmators;

$X$ is a consistent set;

$$
\forall A_{1 \leq k \leq n}\left(A_{k} \in C n T\right)
$$

$T \notin C n X ;$

every sentence belonging to $X$ is considered as true;

every sentence belonging to $X$ represents an amount of empirical evidence;

no empirical counterexample for $T$ ( $\operatorname{such} A$ that $A \in C n T$ and $A$ is false) is known.

The condition (13a) states that confirmations are presented by finite sets of confirmators. Practically, this claim means that only effectively presentable, that is, "small" collections of statements on experiential data provide support for theories. The next condition is obvious and justified by the notorious facts that inconsistent collections of evidence cause attempts to eliminate contradictions. Formula (13c) and (13d) define confirmations are reductive. Formula (13e) also appears as obvious, because particular confirmators are considered as true, even if this assumption is only hypothetical. As far as the issue, concerns (13f) and (13d), I do not assume that empirical evidence (positive as well as negative) is expressed by purely observational, protocol or non-revisable sentences. On the contrary, sentences $A_{1}, \ldots, A_{n}$ confirming $T$ as well as counterexamples for it, can have a theoretical import in the sense that they are proposed as relative to the theory in question or other (e. g. earlier) theoretical systems. No property of induction depends on the assumption that premises of inductive inference are, so to speak, theoretically-free. The only constraint imposed on $A_{1}, \ldots, A_{n}$ as elements of $X$ (see (13a)) and $A$ as a possible counterexample (see $13 \mathrm{~g}$ ) claims that they represent empirical data accessible in the context of $T$. It means that even if the distinction theoretical/empirical cannot be precisely defined in general terms, its concrete instances are sufficiently clear. For example, theoretical categories of classical mechanics appear as empirical data for quantum theory or relatively theory. The idea of absolute empirical protocols, free of any theoretical content, characteristic for early logical empiricism (including Carnap himself) should not be extended as inherently linked with accepting induction as a legitimate epistemological procedure. 
If $X$ is a confirmation of $T$, we can write $T \in C n{ }^{s i n d} X$. This notation does not exclude the situation that more than one theory is inductively supported by the same collection of empirical data, because of the condition (13a). Even more, the same $X$ can support mutually contradictory or contrary theories $T_{j}$ and $T_{k}$, if their intersection is non-empty. However, I will not consider such cases. The next step of analysis consists in an attempt to exhibit formal properties of the operation $C n^{\text {sind }}$. Is it analogous to $C n$ ? The general axioms for $C n$ are the formulas: (i) $X \subseteq C n X$; (ii) $X \subseteq Y \Rightarrow$ $C n X \subseteq C n Y$; (iii) $C n C n X=C n X$; (iv) if $A \in C n$, there is a finite set $Y$ such that $Y \subset X$ and $A \in C n Y$. All these postulates are plausible for $C n^{\text {sind }}$. Firstly, $X \subseteq C n^{\text {sind }} X$, due to (13c); secondly, if $X \subseteq Y$, then $C n^{\text {sind }} X \subseteq C n^{\sin d} Y$ (eventually, we can add that $Y$ is a confirmation of something); thirdly, $C n^{\text {sind }} C n^{\text {sind }} X$ (iteration of confirmation adds nothing new); fourthly, (iv) holds for $C n^{\text {sind }}$, because $X$ is finite by definition (in this case, it is convenient to assume that $T$ (a theory) belongs to $C n^{\text {sind }} X$. The result appears as promising for the first look, because $C n^{\text {sind }}$ deserves to be called a consequence operation. ${ }^{10}$ However, the above mentioned axioms for $\mathrm{Cn}$ do not generate any logic, because they say nothing about logical constants. If we add the deduction theorem as the next postulate, that is, the formula

$$
\text { if } B \in C n(X \cup\{A\}) \text {, then } A \Rightarrow B \in C n X \text {, }
$$

we obtain implicational calculus. In order to justify that (14) holds for $C n^{\text {sind }}$, we must prove that if $B \in C n^{\text {sind }}(X \cup\{A\})$, then $A \Rightarrow B \in C n^{\text {sind }} X$. The antecedent of this implication asserts that $B$ is confirmed by the set $X \cup\{A\}$ of true sentences. However, it is not enough to assert that $B$ is true as well. Consequently, considering the formula $A \Rightarrow B$ as inductively supported by $X$ is not correct. Various proposals can be formulated in order to solve this situation, for instance, introducing the "inductive" implication $A \Rightarrow{ }^{\text {sind }} B$. This solution would require new settings concerning other connectives. Since the expression $A \Rightarrow{ }^{\text {sind }} B$ can be interpreted as "the implication $A \Rightarrow B$ is confirmed to the inductive degree $r$ " (this reading suggests complementing $A \Rightarrow{ }^{\text {sind }} B$ to $A \Rightarrow{ }^{\text {sind }(r)} B$ ), a new definition of the correct implication should be given, for instance, postulating that the degree(of confirmation) of consequent must be equal or greater than the degree of antecedent. Perhaps many-valued or probabilistic logic could be helpful, but I will not enter into this question. Anyway, the numerical value of $r$ or another inductive parameter returns almost at every point of analyzing induction.

As I earlier noted, the definition of the quantitative value attributed to the confirmation of strictly universal hypotheses constitutes a very dramatic problem for the inductive logic. Intuitively speaking, if $H$ is such a hypothesis, its scope of validity is infinite. By definition (13), its confirmation involves a finite amount of data. Since (it is a very informal way of speaking) $\infty-n=\infty$ ( $n$ is a natural number), any confirmation, as defined by (13) does not increase the already achieved degree of inductive support. This argument requires no appeal to other observation (attributed

\footnotetext{
${ }^{10}$ One might complain that monotonicity of $\mathrm{Cn}^{\text {sind }}$ is problematic, because the assumption $X Y$ does not exclude that the set $Y$ contains a counterexample. If we add that $Y$ is a confirmation of something, properties of inductive consequence are less general than $\mathrm{Cn}$.
} 
to Popper) that the initial (or a priori) logical probability of strictly universal hypotheses equals zero. Carnap and other advocates of inductive logic tried to overcome this difficulty by considering universal hypotheses (or laws) as material (theoretical) rules of inference or formal schemata generating sentences concerning particular instances of universal regularities (I omit solutions based on statistics), but this strategy is at odds with scientific realism. Since I share the realistic account of empirical science (more specifically, the view that scientific theories are true or false in semantic models, but I do not enter into this question; see Agazzi 2014 for a defence of this position), I think that instrumentalism in the philosophy of science pays too higher price. Incidentally, the statistical approach to induction opens some possibilities to thinking on confirmation by infinite sequences of events, but, to repeat, it is not logic but applied statistics. Returning to (13), the definition of confirmations does not imply that a theory $T$ must be rejected in the front of counterexamples, because it can be modified in a way or even temporarily neither rejected nor modified (the case of planetary model of atom). In fact, rules of acceptance or rejection of theories are not exhausted by positive (supporting) evidence or negative one (see Hilpinen 1968). For instance, (13) does not take into account the strength of evidence, relative to their intuitively assessed probability or differences between them (the case of general theory of relativity, which had, and still had, a small amount of supporting data, but various and not expected). The researcher can also believe that his theory can be made consistent with anomalous facts (the case of geocentric astronomy). The full account of confirmation certainly exceeds the conditions contained in (13) — this definition concentrates on logical aspects.

\section{Induction and maximally consistent sets}

A construction of maximally consistent set of sentences allows a further analysis of confirmation. This procedure is associated with the Lindenbaum lemma

For every consistent set $X$ of sentences, there is a set $Y$ such that $X \subseteq Y$ and $Y$ is maximally

consistent, that is, if $A \notin Y$, then $Y \cup\{A\}$ is inconsistent

Intuitively, adding a new sentence to a maximally consistent set (the Lindenbaum set), results with inconsistency. The construction of a maximally consistent set proceeds in the following way. Since the set of sentences of any (formal) language $\mathbf{L}$ is countably infinite, we can ascribe a numeral to every sentence of $\mathbf{L}$. Moreover, the infinite sequence of sets $X_{1}, X_{2}, X_{3}, \ldots$ such that (a) $X=X_{0}$; (b) $X_{n+1}=X_{n} \cup\left\{A_{n+1}\right\}$, if $X$ is consistent: (c) $X_{n+1}=X_{n}$, if $X_{n} \cup\left\{A_{n+1}\right\}$ is inconsistent. Now, the union, denote it by $Y$, of sets $X_{1}, X_{2}, X_{3}, \ldots$ is maximally consistent. Informally speaking, if $X$ is consistent, we identify it with $X_{0}$. Then, we take $A_{i}$ and form the set $X_{1}=X_{0} \cup$ $\left\{A_{1}\right\}$, if it is consistent or stay with $X_{0}$, if $X_{0} \cup\left\{A_{i}\right\}$ is inconsistent-this step is "repeated" infinitely number times with respect the further sets; of course, this procedure is infinitary and, thereby, only theoretically. Assume now that we have a theory $T$ and its confirmation $X$. From (13) we have that $X$ is consistent. Thus, by (15), there is a maximally consistent set $Y$ such that $X \subseteq Y$. The latter set contains logical 
consequences of $T$, but also other sentences, which can be entirely irrelevant with respect to support for the theory in question, because they are not $T$-consequences. The extension of confirmations can be described as follows. We assume that $X$ is an initial confirmation of $T$, that is, $X=X_{o}$. Then, we take a formula $A_{1}$ and check whether $A_{1} \in C n T$ or $A_{1} \notin C n T$. Assume that the first eventuality holds. We check whether $A_{1}$ is true. If it is, we form $X_{1}$ as $X \cup\left\{A_{l}\right\}$; if $A_{1}$ is false or contradicts $X$, $X_{1}=X_{0}$. This step is repeated with respect to formulas $A_{2}, A_{3}, \ldots$ Shortly speaking, we construct a sequence of sets $X_{o}, X_{1}, X_{2}, \ldots$ such that $X_{i} \subseteq X_{i+1}(i=0,1,2$, $\ldots$ ) and for every $i, X_{i}$ satisfies the conditions listen in (13). The union of these sets can be regarded as the full consistent set of confirmations for $T$. Denote this set as $\operatorname{CONF}(T)$. Any sentence not belonging to this class is either false or true, but irrelevant as providing the support of $T$. If we add the union $\mathbf{U}$ of sets of the type $X_{i} \cup$ $\left\{A_{i}\right\}$, such that $A_{i}$ is consistent with $X_{i}$ and irrelevant for confirmation of $T$ (for convenience, we include $H_{1}, \ldots, H_{n}$ into $\boldsymbol{U}$ ), the set $\mathbf{C O N F}(T) \cup \mathbf{U}$ is maximally consistent extension of $X$ and, a fortiori, the set $\operatorname{CONF}(T)$. Thus, the full consistent set of confirmations of a theory $T$ is a subset of maximally consistent set of given confirmation of this theory.

The above construction suggests a certain solution of (or at least, a contribution to) the distinction between good and bad induction. Assume that $Z$ is a set consisting of exclusively irrelevant sentences for confirmation of $T$ and that these sentences are true. So $Z$ is consistent. By (15), there exists the Lindenbaum set with respect to $Z$. Now it is evident that there is a serious difference between arguing for $T$ by appealing to $Z$ and by using something from $\operatorname{CONF}(T)$. The set $Z$ does not give reasons for preference $T$ over any other theory $T$, for instance such that $H_{i} \in T$ iff $\neg H_{i} \in T$ '. Informally speaking, a good (valuable) induction selects the content of confirmations in such a way that premises of inductive inference are taken from the full set of confirmators, being the proper subset of the maximally consistent set with respect to $X$ as initially adopted confirmation). However, this statement does not solve the question whether the extension of confirmators-classes provides a better support for theories. More formally, we have

$$
\text { If } X_{i}, X_{i+1} \text { are confirmations of } T \text { and } X_{i} \subset X_{i+1} \text {, then } X_{i+1} \text { confirms } T \text { at least equally as } X_{i} \text {. }
$$

The expression "at least equally" requires two comments. Firstly, "equally" does not refer to quantitative identity, but to a qualitative property. Secondly, the numerical growth of confirmators not necessary leads to a better confirmation. It is related to the earlier mentioned fact that the same (or similar) confirmators have a limited significance for strengthening beliefs that a given confirmation justifies a theory in question. Formula (16) does not depends on introducing $r$ as a numerically defined degree of confirmation. Once again, $\mathbf{C O N F}(T)$ is an infinite sum of confirmations. Hence, extensions of confirmations never achieve $T$, because between it and any set $X_{i}$ occurs infinitely many possible collections of confirmations. We can say that the cardinal degree of completeness of $X_{i}$ (the number of consistent oversystems of this set) is at least infinitely countable. Another expression of this fact is saying that $T$ is the set-theoretical limit of the infinite set of confirmations (see Suszko 1968) in the sense that "almost all" confirmations of $T$ except a finite amount of them are still 
to be constructed. Hence, if we want to prove that $T$ is true, we should effectively define an infinite number of confirmations, but this task i s impossible. However, we can still consider passing from $X_{i}$ to $X_{i+1}$ as important, relatively to stating additional constraints (for instance, the diversity of confirmators or their status as very surprising events, because such a step excludes possible counterexamples. Thus, if we achieve a new confirmation, we can say that our confidence in $T$ actually increases. There is nothing absurd in saying that the confirmation of $T$ is non-zero due to the existence of a conformation set as well as the appearance of a counterexample changes this situation.

How to answer Hume's criticism of the justification of induction? The Humean sceptic would say that passing from $X_{i}$ to $X_{i+1}$ does not contribute to recognizing $T$ as true. Thus, speaking in this way about more and more better confirmation that $T$ is true is only an epistemic persuasion. However, (15) gives a suggestion how to show a weak point of Hume's epistemology by appealing to the classical or semantic concept of truth (the predicate "is true" occurred in my earlier considerations in an intuitive sense). Observe that every confirmation is consistent by definition. The same concerns the full set of confirmations that is $\operatorname{CONF}(T)$. Since, according to the completeness theorem in its semantic version), every consistent set of sentences has a model. Denote the model of $\mathbf{C O N F}(T)$ by $\mathbf{M}^{*}$. All confirmators are true in $\mathbf{M}^{*}$ formally, $A \in \mathbf{C O N F}(T) \Rightarrow A \in \operatorname{VER}\left(\mathbf{M}^{*}\right)$. If $T$ is consistent, it also has a model, let say, $\mathbf{M}^{\mathbf{T}}$. Purely formal considerations suffice to assert that $\mathbf{M}^{*}$ exists and the same concerns $\operatorname{VER}\left(\mathbf{M}^{*}\right)$, but not solve the problem of consistency of $T$. In particular, the consistency of $\mathbf{C O N F}(T)$ does not imply the consistency of $T$-the proof the latter is a separate issue and might be somehow restricted by incompleteness phenomena. In other words, we know that $\mathbf{C O N F}(T)$ has a maximal consistent extension (in fact many such extensions), we cannot infer from this knowledge that $T$ belongs to it. However, (15) gives reasons that if $X_{i}$ is a confirmation, we can rationally (not only by custom or habit as Hume argued) expect that there exists a more comprehensive $X_{i+1}$ as set of truths. Thus, induction seems to be the only way to collect arguments for consistency and truth of $T$. The set VER of truths is compact, that is, its every subset consists of true sentences. Hence, we conclude that every $T$-confirmator is true. The reverse way cannot be conclusive due to the essence of fallible inferences. As a result of the foregoing analysis, we obtain a conclusion that metalogic and formal semantics can contribute to traditional problems associated with inductive inference. ${ }^{11}$ Once again, I strongly stress that I do claim that real processes of empirical confirmation are applications of the Lindenbaum lemma or other metalogical results.

\footnotetext{
11 I do not claim that it is the only way of looking at induction. Another analysis, particularly directed to the justification of induction, is proposed in Schurz (2019). Roughly speaking, it employs game theory and introduces the ideal of optimal inductive strategies.
} 


\section{Popper, Inductivism and Conclusions}

Karl Popper is a symbol of anti-inductivism. His views are negative and positive. Firstly, he criticized induction as (see Popper 1959) as not having any significance for justification of empirical theories. His main argument (I repeat it) against a metrical account of confirmation, points out that the initial (logical) probability of universal hypotheses is close to zero and no further data can change this situation. It seems that all Carnap-like measures of $c(H, e)$ fall under Popper's criticism. Secondly, Popper developed a deductive approach to justification in science (the hypothetico-deductive model of of science), which claims that theories should be falsified (attempted to be shown as false), not verified. We say (see Stegmüller 1977, p. 87)

$X$ is a deductive confirmation of $T$ iff

$T$ is a theory;

$X$ is a set of accepted sentences based on observation;

$T \cup X$ is consistent;

there are two mutually disjoint sets $E$ and $F$ such that (d1) $E \cup F=X$;

$$
F \in C n(T \cup E)
$$

$F$ is a non - empty and exclusively consists of observational sentences accepted as results of severe attempts of rejection of $T$.

The formulation of (17) contains no other linguistic ingredients as general logical terms and epistemological words (acceptance, severe tests). In particular, there is no appeal to degrees of confirmation. Since deductivists, that is Popper and his followers, maintain that the more unsuccessful attempts to falsify a theory, the better confirmation (corroboration-it is technical notion of deductivism) is achieved, the question whether a degree of corroboration (it is the technical notion of deductivism in the discussed context) can be quantitatively ascribed to universal hypotheses appears as legitimate. In the case of positive answer, the worries concerning Carnap's approach return, but if negative one is proposed, the arguments based on maximal consistent sets and (15) might be repeated with respect to falsification, because unsuccessful attempts to prove that $T$ is false, automatically increase our confidence that $T$ is true in $\mathbf{M}^{*}$. So, even if the deductivist reject the view that a quantitative degree of corroboration can be defined, he or she must agree that the qualitative assessment of inductive support is acceptable.

Three conclusions are suggested by the foregoing analysis. Firstly, if induction is conceived as providing a qualitatively assessed support for empirical theories, it can be defended against Hume's objections. Secondly, semantic approach to induction is 
possible. Thirdly, deudctivism in the theory of empirical justifications, appears as a hidden inductivism (see Kotarbińska 1977).

Open Access This article is licensed under a Creative Commons Attribution 4.0 International License, which permits use, sharing, adaptation, distribution and reproduction in any medium or format, as long as you give appropriate credit to the original author(s) and the source, provide a link to the Creative Commons licence, and indicate if changes were made. The images or other third party material in this article are included in the article's Creative Commons licence, unless indicated otherwise in a credit line to the material. If material is not included in the article's Creative Commons licence and your intended use is not permitted by statutory regulation or exceeds the permitted use, you will need to obtain permission directly from the copyright holder. To view a copy of this licence, visit http://creativecommons.org/licen ses/by/4.0/.

\section{References}

Agazzi E (2014) Scientific objectivity and its context. Springer, Cambridge

Ajdukiewicz K (1955) Klasyfikacja rozumowań (Classification of arguments). Stud Logica 2:278-299

Ajdukiewicz K (1958) Zagadnienie racjonalności zawodnych sposobów wnioskowania (The problem of the rationality of non-deductive types of inference). Studia Filozoficzne 4: 14-29; Eng. tr. in Ajdukiewicz K The scientific world-Perspective and other essays 1931-1963. Rediel, Dordrecht 1978:239-253

Ajdukiewicz K (1974) Pragmatic logic. Reidel, Dordrecht

Bandyopadhyay P, Forster MR (eds) (2011) Philosophy of statistics. Elsevier, Amsterdam

Carnap R (1950) Logical foundations of probability. Chicago University Press, Chicago

Cohen LJ, Hesse M (eds) (1980) Applications of inductive logic. Oxford University Press, Oxford

Czeżowski T (2000) The classification of reasoning and its consequences in the theory of sciences. In: Czeżowski T (ed) Knowledge, science and values. A Program for scientific philosophy. Rodopi, Amsterdam, pp 119-133

Gabbay DM, Hartmann S, Woods J (2011) Handbook of the history of logic v 10: Inductive logic. Elsevier, Amsterdam

Hacking I (2002) An introduction to probability and inductive logic. Cambridge University Press, Cambridge

Hájek A, Hitchcock Ch (eds) (2016) Probability and philosophy. Oxford University Press, Oxford

Hilpinen R (1968) Rules of acceptance and inductive logic. North-Holland, Amsterdam

Hume D (1748/2000) An inquiry concerning human understanding. Oxford University Press, Oxford.

Humphreys P (2016) The Oxford handbook of the philosophy of science. Oxford University Press, Oxford

Jeffrey R (1992) Probability and the art of judgment. Cambridge University Press, Cambridge

Johnson G (2017) Argument and inference: an introduction to inductive logic. The MIT Press, Cambridge

Kotarbińska J (1977) The controversy: deductionism vs iductivism. In: Przełęcki M, Wójcicki R (eds) Twenty-five years of logical methodology in Poland. Reidel, Dordrecht, pp 261-278

Kuipers TAF (ed) (2007) Handbook of the philosophy of science. General philosophy of Science-focal issues. Elsevier, Amsterdam

Kyburg HK (1970) Probability and inductive logic. Mamillan, New York

Kyburg HK (1974) The logical foundations of statistical inference. Reidel, Dordrecht

Lakatos I (1968) Changes in the problem of inductive logic. In: Lakatos I (ed) The problem of inductive logic. Noth-Holland, Amsterdam, pp 314-417

Lenzen W (1974) Theorien der Bestätigung wissenschaflicher Hypothesen. Fromman-Holzboog, Stuttgart

Łukasiewicz J (1912). O twórczości w nauce (Creative elements in science). In: Księga pamiqtkowa ku uczczeniu 250-tej rocznicy założenia Uniwersytetu Lwowskieg.o Nakładem Uniwrsytetu Lwowskiego, Lwów: 1-15; Eng. tr. in: Łukasiewicz J, Selected Works. North-Holland, Amsterdam 1970: $1-15$.

Machamer P, Silberstein M (eds) (2002) The Blackwell guide to the philosophy of science. Blackwell, Oxford 
Newell-Smith WH (ed) (2000) A companion to the philosophy of science. Blackwell, Oxford Maher P (1993) Betting on theories. Cambridge University Press, Cambridge

Michalos AC (1971) The Popper-Carnap controversy. Nijhoff, The Hague

Paris J, Vencovská A (2015) Pure inductive logic. Cambridge University Press, Cambridge

Pietarinen J (1972) Lawlikeness, analogy and inductive logic. North-Holland, Amsterdam

Popper K (1959) The logic of scientific discovery. Hutchinson, London

Psillos S (ed) (2013) The Routledge company to philosophy of science. Routledge, London

Schurz G (2019) Hume's problem solved. The MIT Press, Cambridge, Mass

Shafer G (1976) A mathematical theory of evidence. Princeton University Press, Princeton

Stegmüller W (1973) Personelle und statistische Wahrscheinlichkeit. Springer, Berlin

Stegmüller W. (1977) The problem of induction: Hume's challenge and the contemporary answers. In: Stegmüller W (eds) Collected papers on epistemology philosophy of science and history of philosophy. Reidel, Dordrecht, pp 68136

Suszko R (1968) Formal logic and the development of science. In: Lakatos I, Musgrave A (eds) Problems in the philosophy of science. North-Holland, Amsterdam, pp 210-222

Williamson J (2017) Lectures on inductive logic. Oxford University Press, Oxford

Woleński J. (1991) Theories of reasonings in the Lvov-Warsaw School. In: Albertazzi L, Poli R (eds) Topics in Philosophy and Artificial Intelligence. Kulturinstitute der Zentral Europe, Bozen 1991:101-112; repr. in: Woleński J Essays in the History of Logic and Logical Philosophy. Jagiellonian University Press, Kraków 1999, 52-58.

Publisher's Note Springer Nature remains neutral with regard to jurisdictional claims in published maps and institutional affiliations. 